\title{
QUASI-BIRTH-AND-DEATH PROCESSES, LATTICE PATH COUNTING, AND HYPERGEOMETRIC FUNCTIONS
}

\author{
JOHAN S. H. VAN LEEUWAARDEN, ${ }^{*}$ Eindhoven University of Technology and EURANDOM \\ MARK S. SQUILLANTE, ${ }^{* *}$ IBM Thomas J. Watson Research Center \\ ERIK M. M. WINANDS, ${ }^{* * *}$ Eindhoven University of Technology
}

\begin{abstract}
In this paper we consider a class of quasi-birth-and-death processes for which explicit solutions can be obtained for the rate matrix $\boldsymbol{R}$ and the associated matrix $\boldsymbol{G}$. The probabilistic interpretations of these matrices allow us to describe their elements in terms of paths on the two-dimensional lattice. Then determining explicit expressions for the matrices becomes equivalent to solving a lattice path counting problem, the solution of which is derived using path decomposition, Bernoulli excursions, and hypergeometric functions. A few applications are provided, including classical models for which we obtain some new results.
\end{abstract}

Keywords: Quasi-birth-and-death process; matrix-analytic methods; rate matrix; lattice path counting; hypergeometric function

2000 Mathematics Subject Classification: Primary 60J25; 60J05; 60C05; 33C45

Secondary 06B99; 42C10; 60K25; 60J22

\section{Introduction}

Multidimensional Markov processes arise in many fields of science, engineering, and business. The two-dimensional case is of particular theoretical and practical importance, often occurring directly or through decomposition of higher-dimensional processes. In this paper our general interest is in a class of two-dimensional Markov processes, called quasi-birth-and-death (QBD) processes, whose transitions are skip-free to the left and to the right, with no restrictions upward or downward, in the two-dimensional lattice. A wide variety of stochastic models fall within this class, including random walks in the quarter-plane with unit-bounded jumps and many applications in queueing theory.

The invariant distributions of QBD processes, under appropriate conditions, are well known to have a matrix-geometric form. More precisely, the stationary probability vector has a geometric solution in terms of a so-called rate matrix $\boldsymbol{R}$, which is closely related to another matrix, typically denoted by $\boldsymbol{G}$. Together, $\boldsymbol{R}$ and $\boldsymbol{G}$ play a fundamental role in the general theory of matrixanalytic methods. These two matrices are most often obtained by numerically computing the solutions of nonlinear matrix equations, and many algorithms have been developed for doing so.

\footnotetext{
Received 19 September 2007; revision received 6 February 2009.

* Postal address: EURANDOM, PO Box 513, 5600 MB Eindhoven, The Netherlands.

Email address: j.s.h.v.leeuwaarden@tue.nl

** Postal address: Mathematical Sciences Department, IBM Thomas J. Watson Research Center, PO Box 218, Yorktown Heights, NY 10598, USA. Email address: mss@ watson.ibm.com

*** Postal address: Department of Mathematics and Computer Science and Department of Technology Management, Eindhoven University of Technology, PO Box 513, 5600 MB Eindhoven, The Netherlands.

Email address: e.m.m.winands@tue.nl
} 
On the other hand, the probabilistic significance of the $\boldsymbol{R}$ and $\boldsymbol{G}$ matrices has been well established. Hence, in theory, these probabilistic interpretations can be used together with the structural properties of the QBD process to derive explicit expressions for the elements of $\boldsymbol{R}$ and $\boldsymbol{G}$.

The research literature is quite limited with respect to explicit solutions for the $\boldsymbol{R}$ and $\boldsymbol{G}$ matrices. Ramaswami and Latouche [17] explicitly determined $\boldsymbol{R}$ and $\boldsymbol{G}$ in QBD processes for the special cases when the rows of the birth or death transition matrix are proportional to a common row vector, allowing the state space to be infinite in both dimensions. Some of these results were extended much later in [13] to Markov processes of the GI/M/1-type and M/G/1-type. A few studies consider QBD processes with less restrictive transitions, but further restrict attention to the $\boldsymbol{R}$ matrix and to state spaces infinite in only one dimension. Squillante and Nelson [14], [19], [20], [21] exploited the probabilistic interpretation of $\boldsymbol{R}$ to determine explicit solutions for its elements in various stochastic models based on path decomposition and lattice path counting. An explicit description of $\boldsymbol{R}$ for a class of QBD processes, which includes those in [19], [20], and [21], is presented in van Leeuwaarden and Winands [12].

In this paper we investigate a more general class of QBD processes for which we exploit the probabilistic interpretations of $\boldsymbol{R}$ and $\boldsymbol{G}$, and derive solutions for these matrices using path decomposition, Bernoulli excursions, and lattice path counting. Our results are then used to determine explicit expressions for the elements of $\boldsymbol{R}$ and $\boldsymbol{G}$ in terms of hypergeometric functions. These explicit expressions can be used in turn to obtain explicit solutions for the stationary distribution and related quantities of interest. Our approach, which combines various areas of applied probability such as matrix-analytic methods and lattice path counting, is quite general and can be applied to a wide variety of QBD processes.

We make several contributions, the first being the introduction of a class of QBD processes for which the $\boldsymbol{R}$ and $\boldsymbol{G}$ matrices can be explicitly determined. This class includes QBD processes that are infinite in one or both dimensions and that allow less restrictive transitions than in previous studies, thus making the mathematical problem much more intricate and significantly increasing the applicability of the results. Stochastic models that turn out to be members of this class include certain random walks in the quarter-plane and various queueing systems such as the classical longest queue, priority, and feedback models.

A second contribution is that our exact expressions for the fundamental matrix elements provide an explicit characterization of the probabilistic and dynamic behaviors of the stochastic process itself. Moreover, the results derived within our framework for a general class of QBD processes lead to new results for some specific stochastic models, e.g. the longest queue model.

A final contribution is that our results provide an efficient alternative for computing the invariant distribution over those based on numerical algorithms. Moreover, these results may facilitate the implementation and performance of more effective numerical methods. As an example, numerical algorithms for QBD processes that are infinite in both dimensions must be intrinsically based on truncation of one of the dimensions. The explicit results derived in this study can be used to reveal the potential errors induced by such truncations.

In Section 2 we consider a class of homogeneous QBD processes that serve as the initial basis for our analysis. In Section 3 we solve for each element of $\boldsymbol{R}$ and $\boldsymbol{G}$ in terms of counting paths on the two-dimensional lattice, and then in Section 4 we reformulate these solutions in terms of hypergeometric functions. In Section 5 we remove several initial assumptions made to simplify the presentation and discuss various generalizations of our analysis. In Section 6 we then present some classical stochastic models that fall within the introduced class of QBD processes. 


\section{QBD processes}

Consider a continuous-time Markov process $\left\{X(t), t \in \mathbb{R}_{+}\right\}$on the two-dimensional state space $\left\{(n, j): n \in \mathbb{Z}_{+}, j \in\{1, \ldots, M\}\right\}$ that is partitioned as $\bigcup_{n=0}^{\infty} l(n)$, where

$$
l(n)=\{(n, 1),(n, 2), \ldots,(n, M)\},
$$

and $\mathbb{Z}_{+}$and $\mathbb{R}_{+}$denote the nonnegative integer and real numbers. The first coordinate $n$ is called the level and the second coordinate $j$ is called the phase of state $(n, j)$, with the set $l(n)$ referred to as level $n$. Each level may have a finite or infinite number of states, $M$.

This Markov process is called a QBD process when its one-step transitions from each state are restricted to states in the same level or in the two adjacent levels, and a homogeneous QBD process when these transition rates are additionally level independent. The infinitesimal generator $\boldsymbol{Q}$ of the Markov process then takes the block tridiagonal form

$$
\boldsymbol{Q}=\left(\begin{array}{ccccc}
\boldsymbol{B} & \boldsymbol{A}_{0} & & & \\
\boldsymbol{A}_{2} & \boldsymbol{A}_{1} & \boldsymbol{A}_{0} & & \\
& \boldsymbol{A}_{2} & \boldsymbol{A}_{1} & \boldsymbol{A}_{0} & \\
& & \ddots & \ddots & \ddots
\end{array}\right)
$$

where $\boldsymbol{A}_{0}$ and $\boldsymbol{A}_{2}$ are nonnegative matrices, and $\boldsymbol{A}_{1}$ and $\boldsymbol{B}$ have nonnegative off-diagonal elements and strictly negative diagonals. Each of these matrices has dimension $M \times M$.

Let $\pi$ denote the stationary probability vector of this homogeneous QBD process. We shall assume throughout the paper that the QBD process is irreducible and ergodic. Hence, the stationary probability vector exists and is uniquely determined as the solution of $\boldsymbol{\pi} \boldsymbol{Q}=\mathbf{0}$ and $\boldsymbol{\pi} \boldsymbol{e}=1$, where $\boldsymbol{e}$ denotes a column vector of appropriate dimension containing all $1 \mathrm{~s}$. We partition the vector $\pi$ by levels into subvectors $\pi_{n}, n \in \mathbb{Z}_{+}$, where $\pi_{n}$ has $M$ components corresponding to the states of $l(n)$. The matrix-geometric solution of this partitioned stationary probability vector is given by the following theorem.

Theorem 1. Consider a continuous-time $Q B D$ process with infinitesimal generator $Q$ in the form of (1). Suppose that the QBD process is irreducible and ergodic. Then its stationary distribution $\pi$ is given by

$$
\pi_{n}=\pi_{0} \boldsymbol{R}^{n}, \quad n \in \mathbb{N},
$$

where $\boldsymbol{R}$ is the minimal nonnegative solution of the nonlinear matrix equation

$$
\boldsymbol{A}_{0}+\boldsymbol{R} \boldsymbol{A}_{1}+\boldsymbol{R}^{2} \boldsymbol{A}_{2}=\mathbf{0}
$$

with $\operatorname{sp}(\boldsymbol{R})<1$. Furthermore, the stationary probability vector $\boldsymbol{\pi}_{0}$ exists and is uniquely determined by solving the boundary condition

$$
\boldsymbol{\pi}_{0} \boldsymbol{B}+\boldsymbol{\pi}_{1} \boldsymbol{A}_{2}=\boldsymbol{\pi}_{0}\left(\boldsymbol{B}+\boldsymbol{R} \boldsymbol{A}_{2}\right)=\mathbf{0}
$$

and the normalization condition

$$
\sum_{i=0}^{\infty} \boldsymbol{\pi}_{i} \boldsymbol{e}=\boldsymbol{\pi}_{0}(\boldsymbol{I}-\boldsymbol{R})^{-1} \boldsymbol{e}=1,
$$

where I denotes the identity matrix of appropriate dimension. 
This theorem is due to Neuts for the case in which $M<\infty$ (see [15]); the theorem for the case in which $M=\infty$ follows from the results of [23] (see also [11] and [17]) with the spectrum and inverse related to the operator $\boldsymbol{R}$ appropriately defined (see [6], [7], and [8]). From Theorem 1 we know that the stationary distribution is determined once $\boldsymbol{R}$ is obtained. Several iterative algorithms exist for numerically solving (3); an overview of such algorithms is provided in [11].

A related matrix, typically denoted by $\boldsymbol{G}$, also plays an important role together with $\boldsymbol{R}$ in the general theory of matrix-analytic methods. This related $\boldsymbol{G}$ matrix is the minimal nonnegative solution of the nonlinear matrix equation

$$
A_{0} G^{2}+A_{1} G+A_{2}=0
$$

(see [11], [15], and [16]). Some recent algorithms for numerically solving (3) involve first computing the matrix $\boldsymbol{G}$ and then computing the matrix $\boldsymbol{R}$ based on the relationship

$$
\boldsymbol{R}=\boldsymbol{A}_{0}\left(-\left[\boldsymbol{A}_{1}+\boldsymbol{A}_{0} \boldsymbol{G}\right]\right)^{-1}
$$

(see [11]).

\subsection{Probabilistic interpretations}

Our analysis of QBD processes is based on the probabilistic significance of $\boldsymbol{R}$ and $\boldsymbol{G}$. The matrix $\boldsymbol{R}$ records the expected rate of visits to the states of $l(n+1)$ per unit of local time in $l(n)$. More precisely, the element $R_{i j}$ is the expected sojourn time in $(n+1, j)$ before the first return to $l(n)$ given that the process starts in $(n, i)$, expressed in units of the mean sojourn time in $(n, i)$ (see [11] and [15]). Equivalently,

$$
R_{i j}=V_{i j} \frac{\Delta_{i i}}{\Delta_{j j}},
$$

where $V_{i j}$ is the expected number of visits to $(n+1, j)$ before the first return to $l(n)$ given that the process starts in $(n, i)$, and $\boldsymbol{\Delta}=-\operatorname{diag}\left(\boldsymbol{A}_{1}\right)$ so that the fraction in (6) is the ratio of the $i$ th and $j$ th diagonal elements of $\boldsymbol{A}_{1}$.

The matrix $\boldsymbol{G}$ records the probability, starting from $l(n+1)$, of visiting level $l(n)$ in finite time. Suppose that the process is in $l(n+1)$ at time 0 , and define $\tau$ as the first passage time from $l(n+1)$ to $l(n)$. The element $G_{i j}$ then has the probabilistic description

$$
G_{i j}=\mathrm{P}[\tau<\infty, X(\tau)=(n, j) \mid X(0)=(n+1, i)]
$$

(see [11], [15], and [16]). Under our assumption that $\left\{X(t), t \in \mathbb{R}_{+}\right\}$is irreducible and ergodic, it follows that $\tau<\infty$ with probability 1 and, thus,

$$
G_{i j}=\mathrm{P}[X(\tau)=(n, j) \mid X(0)=(n+1, i), \tau<\infty] .
$$

Owing to the homogeneity of $\boldsymbol{Q}$, the elements of $\boldsymbol{R}$ and $\boldsymbol{G}$ do not depend on $n \in \mathbb{Z}_{+}$. We shall exploit the probabilistic interpretations (6) and (7) in our analysis in the next two sections to obtain explicit solutions for $\boldsymbol{R}$ and $\boldsymbol{G}$.

\section{Lattice path counting}

Let us denote by $\left\langle e_{1}, e_{2}\right\rangle$ a one-step transition of the QBD process from state $(n, j)$ to state $\left(n+e_{1}, j+e_{2}\right), n \in \mathbb{N}$, where the probability that this step occurs is denoted by $\varphi\left\langle e_{1}, e_{2}\right\rangle$ and 
obtained straightforwardly from uniformization using $\boldsymbol{A}_{0}, \boldsymbol{A}_{1}, \boldsymbol{A}_{2}$, and $\boldsymbol{\Delta}$. We shall restrict our attention to the class of QBD processes for which the only transitions from state $(n, j), n \in \mathbb{N}$, $j \in\{1, \ldots, M-1\}$, are horizontal steps $\langle-1,0\rangle$ and $\langle 1,0\rangle$, diagonal steps $\langle-1,1\rangle$ and $\langle 1,1\rangle$, and a vertical step $\langle 0,1\rangle$; and the only transitions from state $(n, M), n \in \mathbb{N}$, are horizontal steps $\langle-1,0\rangle$ and $\langle 1,0\rangle$.

The above restrictions imply that, from the states in $l(n), n \in \mathbb{N}$, the QBD process cannot move in the downward direction, and, thus, the matrices $\boldsymbol{R}$ and $\boldsymbol{G}$ are of upper-triangular form. To elucidate the exposition, we further restrict our attention to the case where $M$ is infinite and the diagonal elements of $\boldsymbol{A}_{1}$ are all equal. The former restriction is not prohibitive and its relaxation is discussed in Section 5, whereas the latter simply implies that $R_{i j}=V_{i j}$ such that we would instead use (6) whenever the restriction does not hold. Then, with $R_{h} \equiv R_{i, i+h}$ and $G_{h} \equiv G_{i, i+h}$, we obtain

$$
\boldsymbol{R}=\left(\begin{array}{cccc}
R_{0} & R_{1} & R_{2} & \ldots \\
& R_{0} & R_{1} & \ldots \\
& & R_{0} & \ldots \\
& & & \ddots
\end{array}\right), \quad \boldsymbol{G}=\left(\begin{array}{cccc}
G_{0} & G_{1} & G_{2} & \ldots \\
& G_{0} & G_{1} & \ldots \\
& & G_{0} & \ldots \\
& & & \ddots
\end{array}\right)
$$

\subsection{The $G$ matrix}

We now derive expressions for each element of $\boldsymbol{G}$ in (8) using its probabilistic interpretation. Assume that at time $\tau$ the QBD process undergoes its $\nu$ th transition. In order to calculate the probability in (7), we then need to count all of the paths on the two-dimensional lattice that start in $(n+1, i)$ and end in $(n, i+h)$ after $v$ steps, without visiting any of the states in $l(n)$ prior to the $v$ th transition. Each step of the path will be one of the steps $\langle-1,0\rangle,\langle 1,0\rangle,\langle-1,1\rangle,\langle 1,1\rangle$, and $\langle 0,1\rangle$.

The main idea in deriving a tractable lattice path counting problem is the decoupling of the paths into horizontal and vertical directions.

(i) Consider a path from state $(n+1, i)$ to state $(n, i+h), h \in \mathbb{Z}_{+}$, that consists of $v$ steps and that goes from $l(n+1)$ to $l(n)$ only at the last $v$ th step. Assume that this path includes $s\langle-1,1\rangle$ steps, $u\langle 1,1\rangle$ steps, and, hence, $t=h-s-u\langle 0,1\rangle$ steps.

(ii) We first consider the path in the horizontal direction only. The diagonal steps $\langle-1,1\rangle$ and $\langle 1,1\rangle$ influence both the horizontal and vertical directions. Therefore, we decompose these diagonal steps into

$$
\begin{aligned}
\langle-1,1\rangle & =\langle 0,1\rangle+\langle-1,0\rangle, \\
\langle 1,1\rangle & =\langle 0,1\rangle+\langle 1,0\rangle .
\end{aligned}
$$

(iii) The decomposition of the diagonal steps ensures that the path includes at least $s\langle-1,0\rangle$ steps and $u\langle 1,0\rangle$ steps. Now denote the total number of $\langle 1,0\rangle$ steps by $m$. We then know that the total number of $\langle-1,0\rangle$ steps is $m+1$ (including the $v$ th step). Furthermore, it should hold that $m \geq \max (u, s-1)$.

(iv) The path then consists of a total number of $v=2 m+t+1$ steps, $2 m+1$ of which are in the horizontal direction. When we omit the $v$ th step, the $2 m$ horizontal steps form a Bernoulli excursion (see [22]). The excursion starts in $(n+1, i)$ and consists of $m$ $\langle 1,0\rangle$ steps and $m\langle-1,0\rangle$ steps. Any sequence of steps may occur provided that there are, at each point during the excursion, at least as many $\langle 1,0\rangle$ steps as $\langle-1,0\rangle$ steps; for 


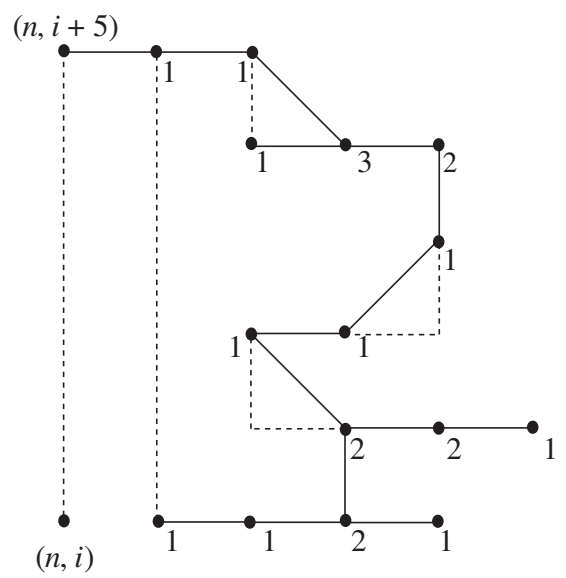

(a) Path from state $(n+1, i)$ to $(n, i+5)$.

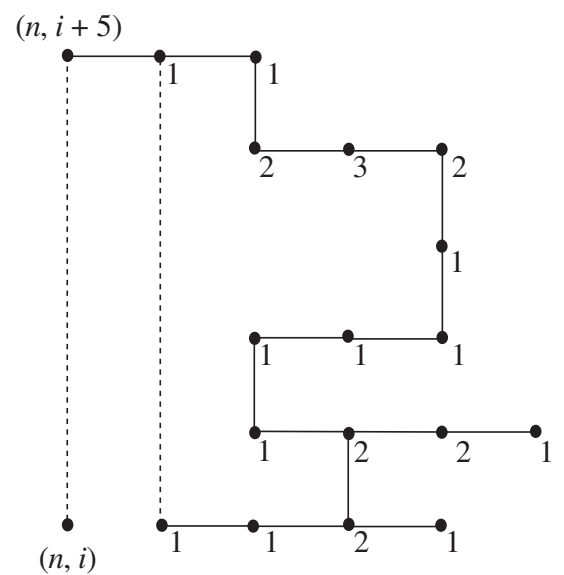

(b) Same path as (a) without diagonal steps.
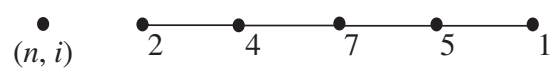

(c) Bernoulli excursion.

FIGURE 1: Example of path decomposition and corresponding Bernoulli excursion.

otherwise, $l(n)$ is visited, and the condition in (i) is violated. The number of possible Bernoulli excursions is given by the $m$ th Catalan number:

$$
\frac{1}{m+1}\left(\begin{array}{c}
2 m \\
m
\end{array}\right) \text {. }
$$

To further elucidate the main idea, let us illustrate the above procedure with an example. In Figure 1(a) we depict a path from state $(n+1, i)$ to state $(n, i+5)$ that consists of $v=21$ steps and that goes from $l(n+1)$ to $l(n)$ only at the $v$ th step. We have indicated in Figure 1(a) the number of times each state is visited. The path includes $s=2\langle-1,1\rangle$ steps, $u=1\langle 1,1\rangle$ step, and $t=2\langle 0,1\rangle$ steps. The diagonal steps are decomposed, leading to the path in Figure 1(b). We then remove the vertical steps to obtain the Bernoulli excursion shown in Figure 1(c). This path is just one out of $\frac{1}{10}\left(\begin{array}{c}18 \\ 9\end{array}\right)=4862$ possible Bernoulli excursions that consist of $2 m=18$ steps. In the above procedure we consider just one path from state $(n, i)$ to state $(n, i+h)$, and reduce it to a Bernoulli excursion.

Let us now change perspective to reflect our original lattice counting problem. Namely, starting from a Bernoulli excursion of length $2 m$, we consider how many paths from state $(n, i)$ to state $(n, i+h)$ can be constructed with $s\langle-1,1\rangle$ steps, $u\langle 1,1\rangle$ steps, and, thus, $t=h-s-u$ $\langle 0,1\rangle$ steps. First, some definitions are needed.

Definition 1. Define $L_{h}(s, u, m)$ to be the number of paths from $(n+1, i)$ to $(n, i+h), h \in \mathbb{Z}_{+}$, that consist of $v=2 m+1+t$ steps and that go from $l(n+1)$ to $l(n)$ only at the $v$ th step. Assume that each path includes $s\langle-1,1\rangle$ steps, $u\langle 1,1\rangle$ steps, and, hence, $t=h-s-u\langle 0,1\rangle$ steps. Define $P_{h}(s, u, m)$ to be the probability of each such path. 
Obviously, we have

$$
P_{h}(s, u, m)=\varphi\langle-1,1\rangle^{s} \varphi\langle 0,1\rangle^{t} \varphi\langle 1,1\rangle^{u} \varphi\langle 1,0\rangle^{m-u} \varphi\langle-1,0\rangle^{m+1-s} .
$$

For $L_{h}(s, u, m)$, we establish the following result.

Lemma 1. Given values of $s$ and $u$ such that $s+u+t=h$, we have

$$
L_{h}(s, u, m)=\frac{1}{m+1}\left(\begin{array}{c}
2 m \\
m
\end{array}\right)\left(\begin{array}{c}
m+1 \\
s
\end{array}\right)\left(\begin{array}{l}
m \\
u
\end{array}\right)\left(\begin{array}{c}
2 m+t \\
t
\end{array}\right) .
$$

Proof. (a) Consider a Bernoulli excursion of length $2 \mathrm{~m}$. We will extend the Bernoulli excursion, which describes the path in the horizontal direction, by the vertical steps to reconstruct a path from state $(n+1, i)$ to state $(n, i+h)$. The vertical steps consist of $s+t+u\langle 0,1\rangle$ steps. However, because of the decomposition in (ii), $s$ steps should be matched to $\langle-1,0\rangle$ steps and $u$ steps should be matched to $\langle 1,0\rangle$ steps. The number of ways to do this is

$$
\left(\begin{array}{c}
m+1 \\
s
\end{array}\right)\left(\begin{array}{l}
m \\
u
\end{array}\right) .
$$

(b) This leaves the placement of the $t$ original $\langle 0,1\rangle$ steps, which can be placed in every state visited by the Bernoulli excursion. This means that we have $2 m+1$ possible states in which the $t\langle 0,1\rangle$ steps can be placed. Note that it is possible for multiple steps to be placed in one state. Therefore, placing the $t\langle 0,1\rangle$ steps is equivalent to distributing $t$ balls over $2 m+1$ bins, and the number of ways to do so is

$$
\left(\begin{array}{c}
2 m+t \\
t
\end{array}\right)
$$

Combining (9), (12), and (13) completes the proof.

Using (7) and $G_{h}=G_{i, i+h}$, we arrive at our main result for the elements of $\boldsymbol{G}$.

Theorem 2. For all $h=0,1, \ldots$, we have

$$
G_{h}=\sum_{s=0}^{h} \sum_{u=0}^{h-s} \sum_{m=\max (u, s-1)}^{\infty} L_{h}(s, u, m) P_{h}(s, u, m),
$$

with $P_{h}(s, u, m)$ as in (10) and $L_{h}(s, u, m)$ as in (11).

To illustrate this result, we return to the example path in Figure 1(a)-(c). Starting from the Bernoulli excursion in Figure 1(c), the path in Figure 1(a) is just one of the $\left(\begin{array}{c}10 \\ 2\end{array}\right)\left(\begin{array}{l}9 \\ 1\end{array}\right)\left(\begin{array}{c}20 \\ 2\end{array}\right)=76950$ paths that can be constructed with $s=2\langle-1,1\rangle$ steps, $u=1\langle 1,1\rangle$ step, and $t=2\langle 0,1\rangle$ steps.

\subsection{The $R$ matrix}

We shall now derive expressions for each element of $\boldsymbol{R}$ in (8) using the probabilistic interpretation in (6). This requires us to determine $V_{i, i+h}$, the expected number of visits to state $(n+1, i+h)$ in the two-dimensional lattice before a return to $l(n)$, given that the process starts in $(n, i)$. The main idea is to decompose the lattice path counting problem into first passage and return visits.

Definition 2. Define $\kappa_{h}$ to be the probability that the QBD process reaches state $(n+1, i+h)$, $h \in \mathbb{Z}_{+}$, before the first visit to $l(n)$, given that the process started in state $(n+1, i)$. 
Definition 3. Define $\gamma$ to be the expected number of visits to state $(n+1, i+h)$ before the first visit to $l(n)$, given that state $(n+1, i+h)$ is reached at least once.

It is then clear that

$$
R_{h}=V_{i, i+h}=\left(\varphi\langle 1,0\rangle \kappa_{h}+\varphi\langle 1,1\rangle \kappa_{h-1}\right) \gamma
$$

We first consider $\gamma$ and suppose that the QBD process enters state $(n+1, i+h)$ for the first time. Then, the path will go through state $(n+1, i+h)$ for a second time (before visiting $l(n))$ when the process moves to $(n+2, i+h)$, starts a Bernoulli excursion of $2 m$ steps $(m=0,1, \ldots)$, and returns from state $(n+2, i+h)$ to state $(n+1, i+h)$. With $\omega=\varphi\langle 1,0\rangle \varphi\langle-1,0\rangle \leq \frac{1}{4}$, the probability that this event happens is given by

$$
\eta=\omega \sum_{m=0}^{\infty} \frac{1}{m+1}\left(\begin{array}{c}
2 m \\
m
\end{array}\right) \omega^{m}=\frac{1-\sqrt{1-4 \omega}}{2}
$$

(see, e.g. [22, p. 561]). Thus, since $\gamma=1+\eta \gamma$, we eventually obtain

$$
\gamma=\frac{2}{1+\sqrt{1-4 \varphi\langle 1,0\rangle \varphi\langle-1,0\rangle}} .
$$

Now turning to the probabilities $\kappa_{h}$, it obviously holds that $\kappa_{0}=1$, and some reasoning yields

$$
\kappa_{h}=\varphi\langle 0,1\rangle \kappa_{h-1}+\varphi\langle 1,0\rangle \sum_{j=0}^{h} G_{h-j} \kappa_{j}+\varphi\langle 1,1\rangle \sum_{j=0}^{h-1} G_{h-j-1} \kappa_{j}, \quad h=1,2, \ldots
$$

Rewriting this expression renders, for $h=1,2, \ldots$,

$$
\kappa_{h}=\frac{\varphi\langle 0,1\rangle \kappa_{h-1}+\varphi\langle 1,0\rangle \sum_{j=0}^{h-1} G_{h-j} \kappa_{j}+\varphi\langle 1,1\rangle \sum_{j=0}^{h-1} G_{h-j-1} \kappa_{j}}{1-\varphi\langle 1,0\rangle G_{0}} .
$$

A combination of (15), (17), and (18) leads to our main result for a description of the elements of $\boldsymbol{R}$.

Theorem 3. For all $h=0,1, \ldots$, we have

$$
R_{h}=\frac{2\left(\varphi\langle 1,0\rangle \kappa_{h}+\varphi\langle 1,1\rangle \kappa_{h-1}\right)}{1+\sqrt{1-4 \varphi\langle 1,0\rangle \varphi\langle-1,0\rangle}}
$$

where $\kappa_{h}$ is as in (18), $\kappa_{0}=1$, and $\kappa_{-1}$ is taken to be 0 .

\section{Hypergeometric functions}

We next express our results for the elements of $\boldsymbol{G}$ and $\boldsymbol{R}$ in terms of hypergeometric functions, which are defined as

$$
F(a, b ; c ; z)=\frac{\Gamma(c)}{\Gamma(a) \Gamma(b)} \sum_{n=0}^{\infty} \frac{\Gamma(a+n) \Gamma(b+n)}{\Gamma(c+n)} \frac{z^{n}}{n !},
$$

where $\Gamma(\cdot)$ is the gamma function. We shall need the following result. 
Lemma 2. For $n=1,2, \ldots$ and $t=1,2, \ldots$,

$$
\Gamma\left(n+\frac{t}{2}\right)=\frac{\sqrt{\pi}}{2^{2(n-1)+t}} \frac{\Gamma(2 n+t-1)}{\Gamma(n+t / 2-1 / 2)} .
$$

Proof. The case in which $t=1$ can be found in [1, pp. 255-256], and is given by

$$
\Gamma\left(p+\frac{1}{2}\right)=\frac{\sqrt{\pi}}{2^{2 p-1}} \frac{\Gamma(2 p)}{\Gamma(p)}, \quad p=1,2, \ldots
$$

For $t=2 m, m=1,2, \ldots$, identity (20) reduces to

$$
\Gamma(n+m)=\frac{\sqrt{\pi}}{2^{2(n+m-1)}} \frac{\Gamma(2(n+m)-1)}{\Gamma(n+m-1 / 2)} .
$$

This yields

$$
\begin{aligned}
\Gamma\left(n+m-\frac{1}{2}\right) & =\frac{\sqrt{\pi}}{2^{2(n+m-1)}} \frac{\Gamma(2(n+m)-1)}{\Gamma(n+m)} \\
& =\frac{\sqrt{\pi}}{2^{2(n+m-1)-1}} \frac{\Gamma(2(n+m-1))}{\Gamma(n+m-1)}
\end{aligned}
$$

which is equivalent to (21) for $p=m+n-1$. In the case in which $t=2 m+1, m=0,1, \ldots$, identity (20) can be written as

$$
\Gamma\left(m+n+\frac{1}{2}\right)=\frac{\sqrt{\pi}}{2^{2(n+m)-1}} \frac{\Gamma(2(n+m))}{\Gamma(n+m)},
$$

which is equivalent to (21) for $p=m+n$. This completes the proof.

We now consider the infinite series in (14):

$$
\sum_{m=\max (u, s-1)}^{\infty} L_{h}(s, u, m) P_{h}(s, u, m)
$$

where $P_{h}(s, u, m)$ and $L_{h}(s, u, m)$ are as in (10) and (11). For the case in which $s-1 \geq u$, we can express (22) in terms of the gamma function (see [1, pp. 255ff.]) as

$$
\frac{\varphi\langle-1,1\rangle^{s} \varphi\langle 0,1\rangle^{t} \varphi\langle 1,1\rangle^{u} \varphi\langle 1,0\rangle^{s-1-u}}{s ! t ! u !} \sum_{m=0}^{\infty} \frac{\Gamma(2 m+2 s+t-1)}{\Gamma(m+s-u)} \frac{\omega^{m}}{m !},
$$

where $\omega=\varphi\langle 1,0\rangle \varphi\langle-1,0\rangle$, as previously defined. From Lemma 2 we obtain

$$
\Gamma(2 m+2 s+t-1)=\frac{2^{2(m+s-1)+t}}{\sqrt{\pi}} \Gamma\left(m+s+\frac{t}{2}\right) \Gamma\left(m+s+\frac{t-1}{2}\right)
$$

and

$$
\frac{2^{2 s-2+t}}{\sqrt{\pi}}=\frac{\Gamma(2 s+t-1)}{\Gamma(s+t / 2) \Gamma(s+(t-1) / 2)} .
$$


Substituting (24) and (25) into the series in (23), and using (19), yields

$$
\begin{aligned}
\sum_{m=0}^{\infty} \frac{\Gamma(2 m+2 s+t-1)}{\Gamma(m+s-u)} \frac{\omega^{m}}{m !} & =\frac{2^{2 s-2+t}}{\sqrt{\pi}} \sum_{m=0}^{\infty} \frac{\Gamma(m+s+t / 2) \Gamma(m+s+(t-1) / 2)}{\Gamma(m+s-u)} \frac{(4)^{m}}{m !} \\
& =\frac{(2 s+t-2) !}{(s-u-1) !} F\left(s+\frac{t}{2}, s+\frac{t-1}{2} ; s-u ; 4 \omega\right),
\end{aligned}
$$

and, hence (suppressing the indices of $L_{h}$ and $P_{h}$ ),

$$
\begin{aligned}
\sum_{m=s-1}^{\infty} L_{h} P_{h}= & \varphi\langle-1,1\rangle^{s} \varphi\langle 0,1\rangle^{t} \varphi\langle 1,1\rangle^{u} \varphi\langle 1,0\rangle^{s-1-u} \\
& \times \frac{(2 s+t-2) !}{s ! t ! u !(s-u-1) !} F\left(s+\frac{t}{2}, s+\frac{t-1}{2} ; s-u ; 4 \omega\right) .
\end{aligned}
$$

In a similar manner, we obtain, for the case in which $u>s-1$ (again suppressing indices),

$$
\begin{aligned}
\sum_{m=u}^{\infty} L_{h} P_{h}= & \varphi\langle-1,1\rangle^{s} \varphi\langle 0,1\rangle^{t} \varphi\langle 1,1\rangle^{u} \varphi\langle-1,0\rangle^{u+1-s} \\
& \times \frac{(2 u+t) !}{s ! t ! u !(u-s+1) !} F\left(u+1+\frac{t}{2}, u+1+\frac{t-1}{2} ; u-s+2 ; 4 \omega\right) .
\end{aligned}
$$

The hypergeometric function $F\left(a+\frac{1}{2}, a ; c ; z\right)$ that emerges in both cases of the infinite series can be rewritten as

$$
\begin{aligned}
F\left(a+\frac{1}{2}, a ; c ; z\right) & =F\left(a, a+\frac{1}{2} ; c ; z\right) \\
& =2^{c-1} \Gamma(c) z^{1 / 2-c / 2}(1-z)^{c / 2-a-1 / 2} P_{2 a-c}^{1-c}\left((1-z)^{-1 / 2}\right),
\end{aligned}
$$

where $P_{v}^{\mu}(\cdot)$ is the Legendre function of the first kind (see [1, p. 562]). Using the relation

$$
P_{v}^{\mu-1}(z)=\frac{P_{v+1}^{\mu}(z)-P_{v-1}^{\mu}(z)}{(2 v+1) \sqrt{z^{2}-1}}
$$

(see [1, p. 334]), we can recursively express the Legendre function $P_{v}^{\mu}$ in terms of Legendre polynomials $P_{v}^{0}$. In turn, these Legendre polynomials have the following explicit form

$$
P_{v}^{0}(x)=\frac{1}{2^{v}} \sum_{m=0}^{\lfloor v / 2\rfloor}(-1)^{m}\left(\begin{array}{c}
v \\
m
\end{array}\right)\left(\begin{array}{c}
2(v-m) \\
m
\end{array}\right) x^{\nu-2 m}
$$

(see [1, p. 775]), which implies that all of the above hypergeometric functions can be computed in a finite number of steps.

\section{Generalizations}

Many assumptions in the foregoing analysis were made to simplify the presentation. Indeed, our results hold for the class of nonhomogeneous QBD processes in which the levels are divided into a nonhomogeneous boundary, comprised of $l(0), \ldots, l(N)$, and a homogeneous portion, comprised of $l(N+1), l(N+2), \ldots, N<\infty$. The generator $\boldsymbol{Q}$ in this case takes the form (1) 
but with the four matrices in the upper leftmost corner replaced by $\boldsymbol{B}_{00}, \boldsymbol{B}_{01}, \boldsymbol{B}_{10}$, and $\boldsymbol{B}_{11}$ having dimensions $N M \times N M, N M \times M, M \times N M$, and $M \times M$. Equations (2), (4), and (5) are then replaced by

$$
\boldsymbol{\pi}_{N+n}=\boldsymbol{\pi}_{N} \boldsymbol{R}^{n}, \quad n \in \mathbb{N}
$$

and

$$
\begin{aligned}
& \left(\boldsymbol{\pi}_{0}, \boldsymbol{\pi}_{1}, \ldots, \boldsymbol{\pi}_{N}\right)\left(\begin{array}{cc}
\boldsymbol{B}_{00} & \boldsymbol{B}_{01} \\
\boldsymbol{B}_{10} & \boldsymbol{B}_{11}+\boldsymbol{R} \boldsymbol{A}_{2}
\end{array}\right)=\mathbf{0}, \\
& \left(\boldsymbol{\pi}_{0}, \boldsymbol{\pi}_{1}, \ldots, \boldsymbol{\pi}_{N-1}\right) \boldsymbol{e}+\boldsymbol{\pi}_{N}(\boldsymbol{I}-\boldsymbol{R})^{-1} \boldsymbol{e}=1 .
\end{aligned}
$$

The number of phases for $l(n), n \in\{0, \ldots, N\}$, can be further generalized to an arbitrary $M_{n}$. Owing to the homogeneous portion of $\boldsymbol{Q}$, the elements of $\boldsymbol{R}$ and $\boldsymbol{G}$ do not depend on $l(N+n)$, $n \in \mathbb{N}$, and are given by the results in Sections 3 and 4 .

Furthermore, our analysis directly applies to discrete-time Markov processes since the probabilistic significance of $\boldsymbol{R}$ and $\boldsymbol{G}$ in this case are $R_{i j}=V_{i j}$ and the discrete-time version of (7) (see [11], [15], and [16]). The restriction of $M=\infty$ does not cause any major technical difficulties and our analysis can be extended to handle the $M<\infty$ case; the technical details are somewhat tedious due to the additional boundary and the different transitions for phase $M$ in each level of the homogeneous portion (since no vertical or diagonal transitions are allowed), and, therefore, are omitted in the present paper. As previously noted, when $\Delta_{i i} \neq \Delta_{j j}$ for one or more $i, j \in\{1, \ldots, M\}$, then our results are straightforwardly adjusted according to (6).

More fundamental extensions of the introduced class of QBD processes are also possible. A critical factor in our analysis is that these QBD processes cannot move downward due to directional restrictions on vertical and diagonal transitions. Similar results may be obtained for classes of QBD processes that cannot move upward (due to the opposite directional restrictions), leftward or rightward (both due to directional restrictions on horizontal and diagonal transitions); see [12] for analogous rotations of a strict subset of these transitions. Although the basic approach may not be fundamentally different from our analysis here, many subtleties need to be addressed and specific probabilistic arguments are required in the analysis of this extended class of QBD processes; these technical details remain for future research. Stochastic models falling in the extended class include a make-to-order/make-to-stock system [3], a two-machine re-entrant line [2], and many more.

\section{Applications}

We end this paper by briefly discussing three classical stochastic models that are members of the introduced class of QBD processes. To simplify the presentation, we primarily focus on symmetric systems in which two queues are served by a single server. Namely, at each queue customers arrive according to a Poisson process with rate $\lambda$ and both customer types require exponentially distributed service times with mean $\mu^{-1}$. The models differ, however, in the way the server capacity is shared among the two queues as explained below. Once again, we stress that many more stochastic models fall within the introduced class.

\subsection{Longest queue model}

In the first model, the server always serves the longest queue, where ties are resolved randomly and the service policy is applied preemptively. This model has been investigated in several studies; see, e.g. [5], [9], and [24]. If $(n, j)$ denotes the state where, in equilibrium, the difference $D=\left|L_{1}-L_{2}\right|$ between the two queue lengths is $n$ and the shortest queue length 
$L^{*}=\min \left(L_{1}, L_{2}\right)$ is $j$, then the QBD queue length process under the single-server longest queue policy is described by the matrices

$$
\boldsymbol{A}_{0}=\left(\begin{array}{ccc}
\lambda & & \\
& \lambda & \\
& & \ddots
\end{array}\right), \quad \boldsymbol{B}_{01}=\left(\begin{array}{cccc}
2 \lambda & & & \\
\mu & 2 \lambda & & \\
& \mu & 2 \lambda & \\
& & \ddots & \ddots
\end{array}\right), \quad \boldsymbol{B}_{00}=\left(\begin{array}{ccc}
-2 \lambda & & \\
& \delta & \\
& & \ddots
\end{array}\right),
$$

and

$$
\boldsymbol{A}_{1}=\boldsymbol{B}_{11}=\left(\begin{array}{lll}
\delta & & \\
& \delta & \\
& & \ddots
\end{array}\right), \quad \boldsymbol{A}_{2}=\boldsymbol{B}_{10}=\left(\begin{array}{cccc}
\mu & \lambda & & \\
& \mu & \lambda & \\
& & \ddots & \ddots
\end{array}\right),
$$

where $N=1$ and $\delta=-(\mu+2 \lambda)$. The multi-server version of this model also falls within the introduced class of QBD processes, where $N$ reflects the number of servers and the nonhomogeneous boundary is appropriately adjusted.

For the single-server and multi-server longest queue policies, where both $t=0$ and $u=0$, (26) significantly simplifies as shown below.

Proposition 1. If $s=0$, we have

$$
\sum_{m=0}^{\infty} L_{h}(0,0, m) P_{h}(0,0, m)=\frac{1-\sqrt{1-4 \omega}}{2 \varphi\langle 1,0\rangle}
$$

and if $s \geq 1$, we have

$$
\sum_{m=s-1}^{\infty} L_{h}(s, 0, m) P_{h}(s, 0, m)=\varphi\langle-1,1\rangle^{s} \varphi\langle 1,0\rangle^{s-1} \frac{(2 s-2) !}{s !(s-1) !}(1-4 \omega)^{1 / 2-s} .
$$

Proof. Equation (28) follows directly from (16). From (26), it follows that

$$
\sum_{m=s-1}^{\infty} L_{h}(s, 0, m) P_{h}(s, 0, m)=\varphi\langle-1,1\rangle^{s} \varphi\langle 1,0\rangle^{s-1} \frac{(2 s-2) !}{s !(s-1) !} F\left(s, s-\frac{1}{2} ; s ; 4 \omega\right) .
$$

The fact that $F\left(s, s-\frac{1}{2} ; s ; 4 \omega\right)=F\left(s-\frac{1}{2}, s ; s ; 4 \omega\right)$, together with the identity $F(a, b ; b ; z)=$ $(1-z)^{-a}$ (see [1, p. 556]), then completes the proof.

\subsection{Priority model}

In the second application we investigate the single-server case where queue 1 has (fixed) priority over queue 2 . More specifically, we consider the preemptive priority rule which implies that type- 1 customers are allowed to interrupt type- 2 customers. This model has a long history; see, e.g. [4] and [10]. Let $(n, j)$ denote the state where, in equilibrium, the lengths of queues 1 and 2 are $n$ and $j$, respectively. The QBD queue length process is described by the matrices

$$
\boldsymbol{A}_{0}=\boldsymbol{B}_{01}=\left(\begin{array}{ccc}
\lambda & & \\
& \lambda & \\
& & \ddots
\end{array}\right), \quad \boldsymbol{A}_{2}=\boldsymbol{B}_{10}=\left(\begin{array}{lll}
\mu & & \\
& \mu & \\
& & \ddots
\end{array}\right),
$$


and

$$
\boldsymbol{A}_{1}=\boldsymbol{B}_{11}=\left(\begin{array}{cccc}
\delta & \lambda & & \\
& \delta & \lambda & \\
& \ddots & \ddots
\end{array}\right), \quad \boldsymbol{B}_{00}=\left(\begin{array}{cccc}
-2 \lambda & \lambda & & \\
\mu & \delta & \lambda & \\
& \ddots & \ddots & \ddots
\end{array}\right),
$$

where $N=1$ and $\delta=-(\mu+2 \lambda)$.

Since both $s=0$ and $u=0$ in the priority model, (27) can be simplified as follows.

Proposition 2. If $s=0$ and $u=0$, we have

$$
\sum_{m=0}^{\infty} L_{h}(0,0, m) P_{h}(0,0, m)=\varphi\langle-1,0\rangle \varphi\langle 0,1\rangle^{t} F\left(1+\frac{t}{2}, 1+\frac{t-1}{2} ; 2 ; 4 \omega\right) .
$$

Proof. The proof follows immediately from (27).

\subsection{Feedback model}

In our final example we consider a single-server, two-queue system with feedback, where queue 1 has preemptive priority over queue 2; see, e.g. [18]. Upon arrival, customers enter queue 1 , and after receiving service, they either depart the system or join queue 2 (with equal probability); in the latter case, customers depart the system after being served at queue 2 . Let $(n, j)$ denote the state where, in equilibrium, the length of queue 1 is $n$ and the number of customers that will eventually ask for service in queue 2 (including those still in queue 1) is $j$. The associated QBD process is described by the matrices

$$
\boldsymbol{A}_{0}=\boldsymbol{B}_{01}=\left(\begin{array}{cccc}
\lambda & \lambda & & \\
& \lambda & \lambda & \\
& \ddots & \ddots
\end{array}\right), \quad \boldsymbol{A}_{2}=\boldsymbol{B}_{10}=\left(\begin{array}{lll}
\mu & & \\
& \mu & \\
& & \ddots
\end{array}\right),
$$

and

$$
\boldsymbol{A}_{1}=\boldsymbol{B}_{11}=\left(\begin{array}{ccc}
\delta & & \\
& \delta & \\
& & \ddots
\end{array}\right), \quad \boldsymbol{B}_{00}=\left(\begin{array}{ccc}
\delta & & \\
\mu & \delta & \\
& \ddots & \ddots
\end{array}\right),
$$

where $N=1$ and $\delta=-(\mu+2 \lambda)$. We again obtain a simplified version of (27).

Proposition 3. If $s=0$ and $t=0$, we have

$$
\sum_{m=u}^{\infty} L_{h}(s, u, m) P_{h}(s, u, m)=\varphi\langle 1,1\rangle^{u} \varphi\langle-1,0\rangle^{u+1} \frac{(2 u) !}{u ! u !}(4 \omega)^{-u-1} B_{4 \omega}\left(1+u, \frac{1}{2}-u\right),
$$

where $B_{x}(\cdot, \cdot)$ is the incomplete beta function defined by (see $\left.[1, p .263]\right)$

$$
B_{x}(a, b)=\int_{0}^{x} t^{a-1}(1-t)^{b-1} \mathrm{~d} t .
$$

Proof. In the case in which $s=0$ and $t=0,(27)$ reduces to

$$
\sum_{m=u}^{\infty} L_{h}(s, u, m) P_{h}(s, u, m)=\varphi\langle 1,1\rangle^{u} \varphi\langle-1,0\rangle^{u+1} \frac{(2 u) !}{(u+1) ! u !} F\left(u+1, u+\frac{1}{2} ; u+2 ; 4 \omega\right) .
$$


The identity

$$
B_{x}(a, b)=\frac{x^{a}}{a} F(a, 1-b ; a+1 ; x)
$$

(see [1, p. 263]) then completes the proof.

\section{References}

[1] Abramowitz, M. And Stegun, I. A. (1964). Handbook of Mathematical Functions with Formulas, Graphs, and Mathematical Tables. US Government Printing Office, Washington, D.C.

[2] Adan, I. J. B. F. And Weiss, G. (2006). Analysis of a simple Markovian re-entrant line with infinite supply of work under the LBFS policy. Queueing Systems 54, 169-183.

[3] AdAN, I. J. B. F. AND VAN DER WAL, J. (1998). Combining make to order and make to stock. OR Spektrum 20, 73-81.

[4] Совнам, A. (1954). Priority assignment in waiting line problems. Operat. Res. 2, 70-76.

[5] Cohen, J. W. (1987). A two-queue, one-server model with priority for the longer queue. Queueing Systems $\mathbf{2}$, 261-283.

[6] Dunford, N. And Schwartz, J. T. (1958). Linear Operators. Part I: General Theory. John Wiley, New York.

[7] Dunford, N. And Schwartz, J. T. (1963). Linear Operators. Part II: Spectral Theory. Self Adjoint Operators in Hilbert Space. John Wiley, New York.

[8] Dunford, N. And Schwartz, J. T. (1971). Linear Operators. Part III: Spectral Operators. John Wiley, New York.

[9] Flatto, L. (1989). The longer queue model. Prob. Eng. Inf. Sci. 3, 537-559.

[10] Jaiswal, N. K. (1968). Priority Queues (Math. Sci. Eng. 50). Academic Press, London.

[11] Latouche, G. and Ramaswami, V. (1999). Introduction to Matrix Analytic Methods in Stochastic Modeling. Society for Industrial and Applied Mathematics, Philadelphia, PA.

[12] Van Leeuwaarden, J. S. H. And Winands, E. M. M. (2006). Quasi-birth-and-death processes with an explicit rate matrix. Stoch. Models 22, 77-98.

[13] LiU, D. AND ZhaO, Y. Q. (1997). Determination of explicit solutions for a general class of Markov processes. In Matrix-Analytic Methods in Stochastic Models (Lecture Notes Pure Appl. Math. 183), Dekker, New York, pp. 343-357.

[14] Nelson, R. D. and Squillante, M. S. (1996). Stochastic analysis of affinity scheduling and load balancing in parallel processing systems. In Proc. 5th INFORMS Computer Science Technical Section Conference on Computer Science and Operations Research: Recent Advances in the Interface (January 1996).

[15] Neuts, M. F. (1981). Matrix-Geometric Solutions in Stochastic Models. Johns Hopkins Press, Baltimore, MD.

[16] Neuts, M. F. (1989). Structured Stochastic Matrices of M/G/1 Type and Their Applications. Marcel Dekker, New York.

[17] Ramaswami, V. and Latouche, G. (1986). A general class of Markov processes with explicit matrix-geometric solutions. OR Spektrum 8, 209-218.

[18] Schrage, L. E. (1967). The queue M/G/1 with feedback to lower priority queues. Management Sci. 13, $466-474$.

[19] Squillante, M. S. (1998). Matrix-analytic methods in stochastic parallel-server scheduling models. In Advances in Matrix Analytic Methods for Stochastic Models, Notable, New Jersey, pp. 311-340.

[20] SQuillante, M. S. (2005). Stochastic analysis of resource allocation in parallel processing systems. In Computer System Performance Modeling in Perspective: A Tribute to the Work of Prof. K. C. Sevcik, Imperial College Press, London, pp. 227-256.

[21] Squillante, M. S. and Nelson, R. D. (1991). Analysis of task migration in shared-memory multiprocessors. In Proc. ACM SIGMETRICS (May 1991), ACM Press, New York, pp. 143-155.

[22] TAKács, L. (1991). A Bernoulli excursion and its various applications. Adv. Appl. Prob. 23, 557-585.

[23] Tweedie, R. L. (1982). Operator-geometric stationary distributions for Markov chains, with application to queueing models. Adv. Appl. Prob. 14, 368-391.

[24] Zheng, Y. And ZIPKIN, P. H. (1990). A queueing model to analyze the value of centralized inventory information. Operat. Res. 38, 296-307. 